\title{
Shocks in sand flowing in a silo
}

\author{
Azadeh Samadani $^{1}$, L. Mahadevan ${ }^{2}$, A. Kudrolli ${ }^{1}$ \\ 1 Department of Physics, Clark University, Worcester, MA 01610, USA \\ ${ }^{2}$ Department of Applied Mathematics and Theoretical Physics, Cambridge University, CB3 9EW, UK
}

(November 12, 2018)

\begin{abstract}
We study the formation of shocks on the surface of a granular material draining through an orifice at the bottom of a quasi-two dimensional silo. At high flow rates, the surface is observed to deviate strongly from a smooth linear inclined profile giving way to a sharp discontinuity in the height of the surface near the bottom of the incline, the typical response of a choking flow such as encountered in a hydraulic jump in a Newtonian fluid like water. We present experimental results that characterize the conditions for the existence of such a jump, describe its structure and give an explanation for its occurrence.
\end{abstract}

The flow of granular materials presents a multitude of experimental and theoretical challenges in many-body physics, as manifested in the unusual collective behavior of a large assembly of macroscopic grains that interact with each other through collisions and friction. The phenomena of interest in quasi-static motion range from jamming transitions, the formation and evolution of shear bands, and the transmission of forces and acoustic signals in granular packing [1 3]. In the dynamical regime associated with technological applications such as silo flows, other questions of interest include the origin and maintenance of fluctuations and their relation to mean flows in the bulk, and the nucleation and evolution of avalanche like behavior in the vicinity of the surface 19 . 6 .

A system of long-standing technological interest in this last situation is associated with flow in a silo. Building on an understanding of simple properties such as the flow rate as a function of the draining orifice size and the shape of the silo [1], recent work has focused on the details such as the form of the velocity profile of the bulk flowing regions. In [7] we studied the flow in the vicinity of the orifice of a silo. The predominant flow is restricted to a parabolic region centered at the origin and may be interpreted in terms of simple diffusion models [8.96. Here, we complement those studies by considering the dynamical evolution of the form of the free-surface in a quasi two-dimensional silo flow.

Our system consists of a flat-bottomed silo that is quasi-two-dimensional: $89 \mathrm{~cm}$ wide, $45 \mathrm{~cm}$ high and 2.54 $\mathrm{cm}$ deep, the last being between 25-1000 times the typical grain size. The orifice itself has a square cross-section of width equal to that of the silo, with an attached valve to control the flow rate $Q$ (see Fig. 1). We use spherical glass grains with diameters $1 \mathrm{~mm}, 500 \mu \mathrm{m}, 100 \mu \mathrm{m}$, and $30 \mu \mathrm{m}$ to study the dependence of the phenomenon on grain size.

The surface and the bulk of flow is visualized through the glass side walls of the silo using two types of CCD cameras. The Kodak ES 1.0 digital camera with a resolution of 1000 pixels $\times 1000$ pixels with a maximum frame rate of 30 per second was used to measure the sur- face profile and to determine the location of the static and mobile regions inside the silo. A second high speed Kodak camera with a maximum frame rate of 1000 per second and resolution of $256 \times 240$ pixels was used to measure the velocity of the particles. By focusing in small regions where a particle corresponds to 3-4 pixels, we are able to identify and track individual particles over consecutive frames with an accuracy of $<0.1$ pixels and thus determine the velocity.

When the orifice is opened, bulk flow is rapidly established and the free surface initially subsides as an inverted Gaussian and then quickly develops in to a V shape. The angle of inclination of the surface approximately corresponds to the angle of repose of the glass beads. For low flow rates, the free-surface has a constant slope, as shown in Fig. 2(a). However as the flow rate is increased past a threshold by opening the valve, the inclined free-surface is no longer linear but shows a region of abrupt height transition right above the orifice, as shown in Fig. 2(b).

This unusual surface feature is similar to a hydraulic jump in a Newtonian fluid observed when a stream of liquid encounters an obstacle (such as observed when water impinges onto a flat plate). Past work 10 12] has shown that shock-like features similar to hydraulic jumps are observable in granular flows in the presence of an externally introduced physical obstacle such as a vertical plate in a channel flow. In these studies it was noted that the the ratio of inertial force to the gravitational force in the system given by the Froude number exceeds one, in analogy to the hydraulic jump observed in a fluid. Although, in granular flow it is less obvious that this is important as in fluids where it corresponds to the flow velocity exceeding the wave speed for shallow depths. This fact was also noted earlier [11].

In the case of the silo flow treated here, the jump is spontaneously generated by the flowing grains which creates its own "obstacle" by choking right above the orifice. Moreover, unlike in a Newtonian fluid or in the channel flows of granular materials where the entire fluid is in motion, here the grains exist in different "phases" in different regions; away from the orifice and the free-surface, 
they barely move at all, in the neighborhood of the orifice they move slowly, near the free-surface they flow like liquids, and veritably boil into a gas in the vicinity of the free-surface shock [Fig. 2(c)]. To understand the formation of the free-surface shock it is important to understand the nature of the granular flow below the surface. We locate the surface and the boundary between the mobile and static regions as the silo empties using the high resolution digital camera. Two temporally separated images are subtracted to determine the boundary between static and mobile regions. This boundary along with the surface is shown for one-half of the silo in Fig. 3 as the granular matter discharges.

Since all the grains must eventually exit through the orifice, the vertical velocity $v(x, y)$ is largest there, and the horizontal velocity $u(x, y)$ is smallest. However, as one moves away in the vertical direction $v$ gets smaller, while $u$ correspondingly becomes larger, and the extent of the flowing region becomes larger. As voids diffuse upwards from the orifice, they explore a larger and larger distance in the horizontal direction, and the bulk flow seems to be driven by diffusive motion. In fact, the flow in the silo close to the orifice can be described remarkably well in terms of an invariant boundary between flowing and non-flowing regions of the form $y \sim x^{2} / d$ with $d$ being the grain diameter [7], consistent with the kinematic model for silo flows [8].

The flow near the free surface outside this parabolic region is confined to a shallow layer where particles move essentially parallel to the surface. However above the orifice, the flow near the free surface changes from predominantly along the surface to a bulk flow towards the orifice. The size of this intermediate region is a function of the height $h$ of the free surface above the orifice; this region is very large at early times, and gets progressively smaller. We parameterize this intermediate region using the distance $l$ from the bottom of the surface to the point where the flow can be described by the parabola (dashed line in Fig. 3). The inset to Fig. 3 shows $l$ as a function of $h$. Using the high speed camera, we measured the velocity of particles $v_{s}(s, z)$ in the neighborhood of four equidistant locations along the free surface, with $s_{i}, i=1-4$ indicated in Fig. 3 as a function of the depth $z$ perpendicular to the surface. In Fig. 4 , we plot $v_{s}\left(s_{i}, z\right)$ vs. $z$ on a $\log$-linear scale. The velocity decays rapidly for the shallow flowing regions $\left(s_{1}, s_{2}\right)$ and is described by $v_{s}(s, z)=v_{0} \exp \left(-\alpha_{1} z / d-\alpha_{2}(z / d)^{2}\right)$ where $\alpha_{1}$ and $\alpha_{2}$ are dimensionless quantities that depend on the location of the surface. In the intermediate region $\left(s_{3}, s_{4}\right)$, the velocity profile can be described by a single exponential $v_{s}(s, z)=v_{0} \exp \left(-\alpha_{1} z / d\right)$. Fig. A also shows that the particles quickly reach a steady velocity characterized by the balance between gravitational forcing and inelastic collisions [13].

We followed the free-surface as a function of time using the high resolution camera (Fig. 5) while simultaneously measuring $v_{0}$ (Fig. 6) for the $100 \mu \mathrm{m}$ glass beads. In Fig. 6, we see that $v_{0}$ increases as the silo empties. For low $Q$, the surface always remains $\mathrm{V}$ shaped throughout the draining process. For larger $Q$ the $\mathrm{V}$ shaped surface changes into a $U$ shaped surface eventually leading to the formation of a granular jump. For $100 \mu \mathrm{m}$ beads the shock appears at a height of about $10 \mathrm{~cm}$ for a $Q$ of 250 $\mathrm{g} \mathrm{s}^{-1}$, and at a height of about $6 \mathrm{~cm}$ for a $Q$ of $170 \mathrm{~g} \mathrm{~s}^{-1}$. A shock is not observed if the $Q$ is decreased further by using the valve. Thus the shock appears only when the surface velocity exceeds a critical value and occurs at a higher height for higher $Q$ (dashed line in Fig. 6).

We also used beads of various sizes to study its effect on the appearance of the shock. It has been proposed that the flow rate $Q \propto(W-\alpha d)^{5 / 2}$, where $W$ is the width of the silo, and $\alpha$ is a constant between 0 and 1 depending on the geometry of the grain with size $d$ [4. However, we observe that the size of particles has a greater influence on the flow rate than accounted for by this formula. The maximum $Q$ for $30 \mu \mathrm{m}, 100 \mu \mathrm{m}, 500 \mu \mathrm{m}$ and $1 \mathrm{~mm}$ beads was $260 \mathrm{~g} \mathrm{~s}^{-1}, 250 \mathrm{~g} \mathrm{~s}^{-1}, 230 \mathrm{~g} \mathrm{~s}^{-1}$ and $200 \mathrm{~g} \mathrm{~s}^{-1}$ respectively for the same $(2.54 \mathrm{~cm} \times 2.54 \mathrm{~cm})$ orifice. In case of $30 \mu \mathrm{m}$ beads, the shock appears when the surface is approximately $30 \mathrm{~cm}$ above the orifice as opposed to the $10 \mathrm{~cm}$ for $100 \mu \mathrm{m}$. In case of $1 \mathrm{~mm}$ beads, a clear shock does not develop although a stagnation zone at the base of the incline surface is observed. We plot the critical height $h_{c}$ versus the flow rate for various $d$ in Fig. 7 to characterize the transition of the surface from V-shape to that where a jump is observed. We note that the ratio of the drag force to weight of the particles $m g$, is less than $0.1 \%$ for $1 \mathrm{~mm}$ particles and less than $1 \%$ for 100 $\mu \mathrm{m}$ and therefore negligible. Therefore we believe that it is not the cause of the increase in height. The drag force for $30 \mu \mathrm{m}$ particles is about $10 \%$ and may be important. The previous experiments [7] for $2.54 \mathrm{~cm}$ and $1.27 \mathrm{~cm}$ wide silos, showed that the wall friction does not significantly add to the drag force experienced by the particles as measured by the angle of repose and the shape of the parabolic flow region and therefore is negligible.

To rationalize these observations, we start by considering the increase in $v_{0}$ which occurs for essentially geometric reasons. The flow rate $Q$ is proportional to the grain flux past the dashed lines shown in Fig. 3, and scales as $W \times \int v_{s}(s, z) d z$, where $W$ is the width of the silo. Using the observed exponential dependence of $v_{s}(s, z)$, $Q \sim v_{0} \times h_{0}$. As the silo empties, the bulk flow is confined to a smaller and narrower region (see Fig. 3). We have observed $Q$ by measuring the mass of the granular matter flowing out of the orifice in a fixed interval of time and find that $Q$ is constant to within 5\% [14. Since $Q$ is constant, $v_{0}$ must increase with time in the intermediate region whose extent simultaneously decreases. Therefore the transition from surface to bulk flow becomes more and more abrupt. This increases the frequency of inelastic collisions in the neighborhood of the valley leading to 
a region of lower mean velocities (see Fig. 2(c)). The decrease in the horizontal component of the velocity as the grains approach the center of the silo leads to the conditions for a kinematic shock as faster grains pile up behind (and atop) the slower grains. Owing to the symmetry of the flow from either side, the free surface responds by first flattening along its axis of symmetry (see Fig. 5). As $v_{0}$ exceeds a critical value, an abrupt increase in the height of the free surface is the only recourse for the rapidly flowing surface grains which form a granular jump.

In case of collisional granular flows, theoretical approaches have been developed first by [15] and [16] using mass-momemtum-energy-conservation to derive continuum equations. These approachs and subsequent improvements have been also reviewed by [17]. However, applying these continuum approachs, especially in case of free boundary conditions as we have on one side of the flow and enduring contacts at the bottom of the surface flow, is extremely difficult. Therefore in the following we attempt scaling arguments to explain the phenomena.

To quantify the criterion for the formation of the jump, we consider the analogous case of a hydraulic jump in a fluid which occurs when the velocity of flow exceeds the propagation speed of a surface perturbation. Dimensional arguments lead to an estimate of the speed of propagation of surface perturbations as $v_{p} \sim \sqrt{g D}$, where $D$ corresponds to the depth of the flow 18]. In our case $D \sim 20 \times d$. Therefore $v_{p} \sim 14 \mathrm{~cm} \mathrm{~s}^{-1}$ for 100 $\mu \mathrm{m}$ beads, in qualitative agreement with the surface velocity at which the shock is observed to occur. Finally, because $v_{p}$ increases with $d$, this is also consistent with the occurrence of the shock at a much higher height for $30 \mu \mathrm{m}$ particles and the lack of a shock in case of the larger $1 \mathrm{~mm}$ beads.

In conclusion, we have shown that a dynamic jamming event in silo flows can lead to the formation of free surface shocks that are similar to hydraulic jumps in simple fluids whose flow is obstructed. An interesting feature of the granular jump is that the obstruction is generated spontaneously by the flowing grains. We have given a kinematic explanation for our observations, but in the absence of an elaborate theory that describes granular flows, a quantitative explanation remains an open question.

Acknowledgments We thank D. Blair, E. Weeks, and W. Losert for fruitful discussion. This work was supported by the National Science Foundation under grant number DMR-9983659 (Clark), the donors of the Petroleum Research Fund (Clark) and the Office of Naval Research through an NYI award (LM). AK also thanks the Alfred P. Sloan Foundation for its support; LM also thanks ENS-Paris and ESPCI for support via the Chaire Condorcet and Chaire Paris Sciences.
[1] Jaeger, H., Nagel, S. R., and Behringer, R., Rev. Mod. Phys. 68, 1259(1996).

[2] Rajchenbach, J., Adv. Phys 49, 229 (2000).

[3] Liu, A. J., and Nagel, S. R., Nature (London) 396, 21 (1998).

[4] Nedderman, R. M., Statics and kinematics of granular materials, Cambridge (1992).

[5] Bouchaud, J.-P., Cates, M., Raviprakash, J., and J. Edwards, J. Phys. I (France) 4, 1383 (1994).

[6] de Gennes, P. G., Rev. Mod. Phys. 71, S374 (1999).

[7] Samadani, A., Pradhan, A., and Kudrolli, A., Phys. Rev. E 60, 7203 (1999).

[8] Nedderman, R. M., and Tuzun, U., Powder Tech. 22, 243 (1979).

[9] Mullins, W. W., Powder Tech. 9, 29 (1974).

[10] Morrison, H. L., and Richmond, O., J. Appl. Mech. 43, 49 (1976).

[11] Savage, S. B., J. Fluid Mech. 92, 53 (1979).

[12] Brennen, C. E., Sieck, K. and Paslaski, J., Powder Tech. 35, 31 (1983).

[13] An exponentially decaying velocity profile as a function of depth has been reported recently in surface flows 19,20 and in a Couette geometry 21. However stretchedexponential profiles have been also reported in a Couette geometry 22]. Our data appears to be more consistent with a stretched exponential than a simple exponential function in the shallow surface flow regime.

[14] We note that during the ultimate stages of draining, the sand empties like a Newtonian fluid under its own hydrostatic head; this could be a potential choking mechanism but in our experiments the shock typically forms much earlier, and therefore must have its origins elsewhere.

[15] Haff, P. K., J. Fluid Mech. 134, 401 (1983) .

[16] Jenkins, J., and Savage, S., J. Fluid. Mech. 130, 187 (1983).

[17] Campbell, C. S., Annu. Rev. Fluid Mech. 22, 57 (1990).

[18] Mahadevan, L., and Pomeau, Y., Europhys. Lett. 46, 595 (1999).

[19] Komatsu,T. S., Inagaki, S., Nakagawa, N., and Nasuno, S., Phys. Rev. Lett. 86, 1757 (2001).

[20] Lemieux, P.-A., and Durian, D. J., Phys. Rev. Lett. 85, 4273 (2000).

[21] Losert, W., Bocquet, L., Lubensky, T. C., and Gollub, J. P., Phys. Rev. Lett. 85, 1428 (2000).

[22] Mueth, D. M., Debregeas, G. F., Karczmar, G. S., Eng, P. J., Nagel, S. R., Jaeger, H. M., Nature (London) 406, 385 (2000). 


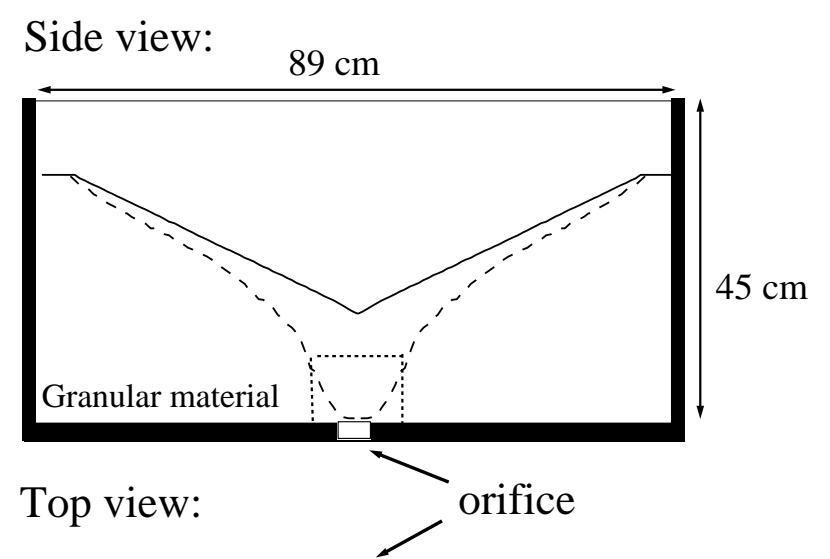

$2.54 \mathrm{~cm}$

FIG. 1. The experimental apparatus is a quasi-two dimensional silo with glass side walls. The dashed curve indicates an example of the boundary between rapidly flowing and static regions inside the silo. The dotted square box close to the orifice shows the approximate area corresponding to the images shown in Fig. 2(a) and Fig. 月(b).

FIG. 2. (a) Image of granular matter draining from an orifice inside a quasi-two dimensional silo as a function of time. The surface is linear in this case. $Q \sim 50 \mathrm{~g} \mathrm{~s}^{-1}, d=100 \mu \mathrm{m}$. The images correspond to a $7 \mathrm{~cm} \times 7 \mathrm{~cm}$ region close to the orifice. (b) The surface is observed to show an increase in height similar to a hydraulic jump when a rapidly flowing fluid in a channel encounters an obstacle. $Q=200 \mathrm{~g} \mathrm{~s}^{-1}$. (c) Closeup image $(\times 3)$ of the shock as it is forming. The duration of the exposure is $4 \mathrm{~ms}$ and therefore the fast moving particles appear as streaks. By comparison, the particles inside the jump appear almost frozen. A movie of the jump can be viewed at http://physics.clarku.edu/ akudrolli/shock.html.

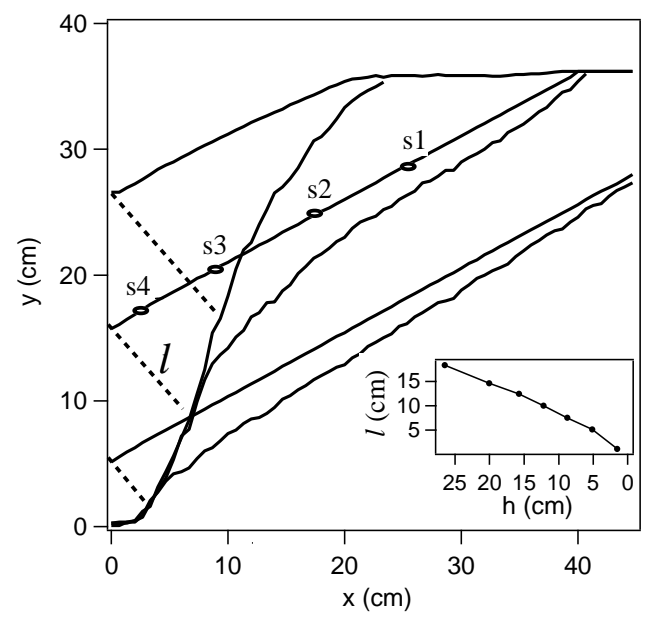

FIG. 3. The surface and the boundary between flowing and static regions at three different times. The flow region is parabolic, and stay parabolic near the orifice during discharge. The orifice is centered at the origin $(0,0)$. The dashed lines show the size of the intermediate region. The points $\mathrm{s} 1$, s2, s3, and s4 mark the locations where the velocity is measured and plotted in Fig. A. $d=1 \mathrm{~mm}$. Inset: The length $l$ that measures the size of the intermediate region as a function of height $h$ of the surface above the orifice is observed to decrease as the silo empties.

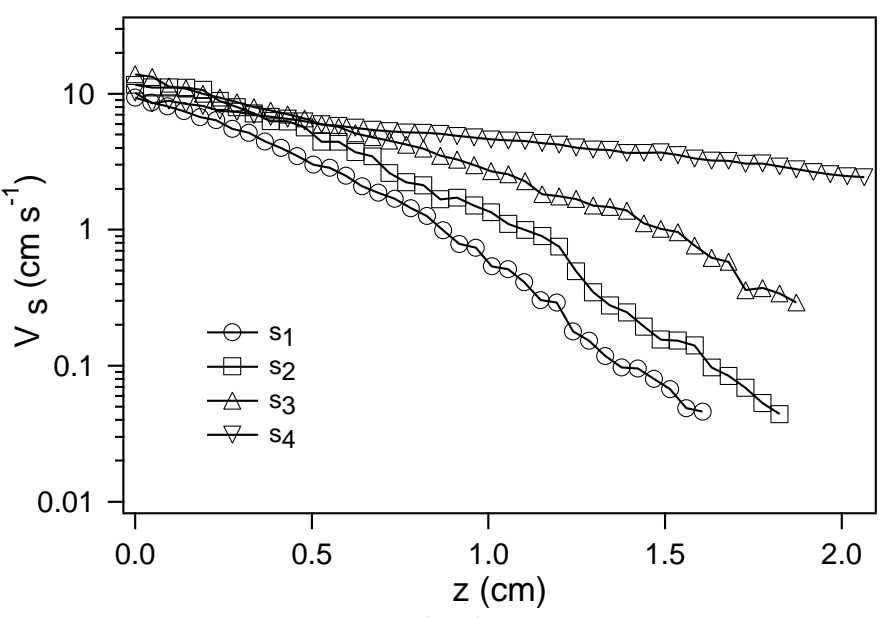

FIG. 4. The velocity $v_{s}(s, z)$ as a function of depth $z$ at four positions $s_{1}-s_{4}$ along the surface (indicated in Fig. 3). $d=1 \mathrm{~mm}$. The $s$-axis is chosen parallel to the surface and the $z$ axis is chosen perpendicular to the surface and pointing inwards. The velocities are observed to decay approximately exponentially from the surface. The length over which the velocity decays $h_{0}$ is approximately $10-20$ grain diameters. Note also that the particles have reached an approximately constant velocity $v_{s}(s, 0)=v_{0}$ along the surface.

FIG. 5. The surface profile of the granular matter draining from a silo as a function of time showing the development of the jump. $\left(Q \sim 170 \mathrm{~g} \mathrm{~s}^{-1}, d=100 \mu \mathrm{m}\right)$. The origin is located at the center of the orifice. The first four surface profiles are separated by 5 second time intervals, to show the features of the jump, the last five are separated by 3 second time intervals. The dashed lines are guides to the eye to compare the surface profile to that at low $Q$. The surface flattens and then a shock is observed to develop. 


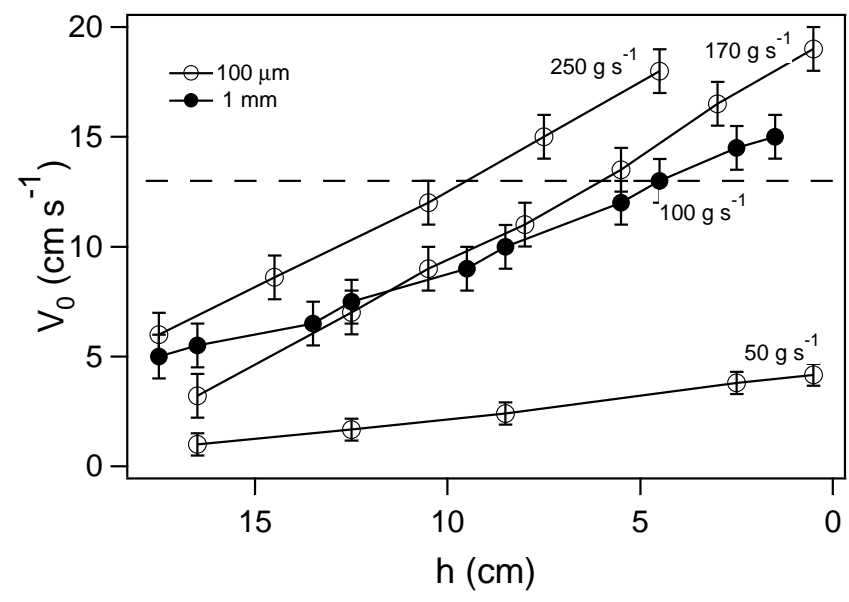

FIG. 6. The velocity of grains $v_{0}$ at the surface as a function of height above the orifice for $d=100 \mu \mathrm{m}$ and $d=1 \mathrm{~mm}$ glass beads. The velocities are measured at a position just before the location where the jump occurs $\left(s_{4}\right.$ in Fig. 3). The dashed line corresponds to the estimate of the critical velocity of $14 \mathrm{~cm} \mathrm{~s}^{-1}$ for 100 micron particles. The critical velocity for $1 \mathrm{~mm}$ particles is approximately $44 \mathrm{~cm} \mathrm{~s}^{-1}$.

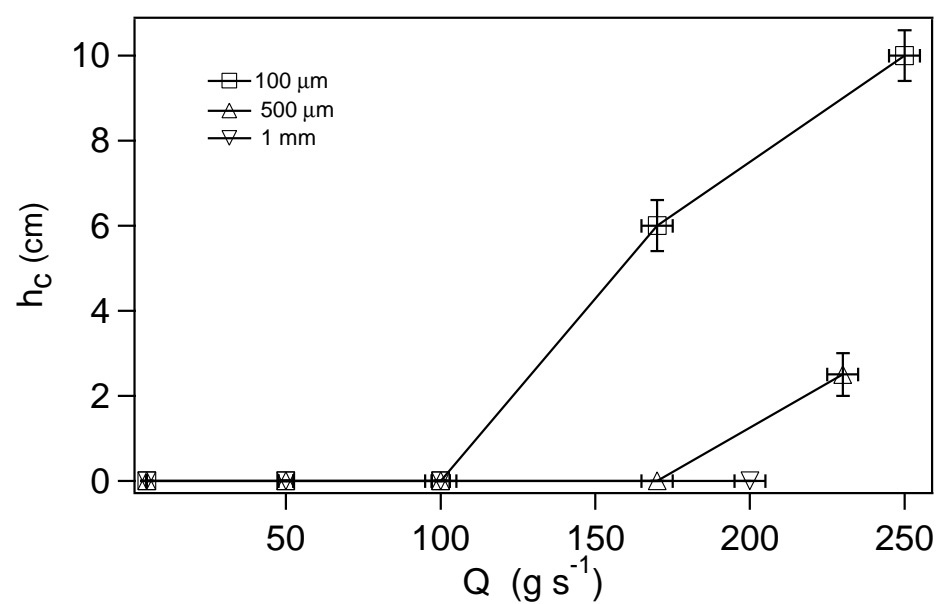

FIG. 7. A plot of the critical height $h_{c}$ where the jump first appears as a function of the flow rate $Q$ for glass particles of various diameters $d$. A clear jump is not observed for $1 \mathrm{~mm}$ particles athough a small stagnant region is observed at the fastest flow rate. 
This figure "fig2.jpg" is available in "jpg" format from: http://arxiv.org/ps/cond-mat/0110427v1 
This figure "fig5.jpg" is available in "jpg" format from: http://arxiv.org/ps/cond-mat/0110427v1 